\title{
LOCAL MINIMIZERS OF FUNCTIONALS WITH MULTIPLE VOLUME CONSTRAINTS*
}

\author{
Édouard Oudet ${ }^{1}$ AND Marc Oliver Rieger ${ }^{2}$
}

\begin{abstract}
We study variational problems with volume constraints, i.e., with level sets of prescribed measure. We introduce a numerical method to approximate local minimizers and illustrate it with some two-dimensional examples. We demonstrate numerically nonexistence results which had been obtained analytically in previous work. Moreover, we show the existence of discontinuous dependence of global minimizers from the data by using a $\Gamma$-limit argument and illustrate this with numerical computations. Finally we construct explicitly local and global minimizers for problems with two volume constraints.
\end{abstract}

Mathematics Subject Classification. 49J, 65K10

Received November 19, 2006. Revised June 14, 2007.

Published online February 7, 2008.

\section{INTRODUCTION}

Let $\Omega$ be a bounded open set in $\mathbb{R}^{n}$. The general form of a variational problem on $\Omega$ with two level set constraints is given by the minimization of

$$
\begin{array}{r}
\text { Minimize } E(u):=\int_{\Omega} f(x, u(x), \nabla u(x)) \mathrm{d} x, \\
|\{x \in \Omega, u(x)=a\}|=\alpha, \\
\\
|\{x \in \Omega, u(x)=b\}|=\beta,
\end{array}
$$

where $u \in H^{1}(\Omega)$ and $\alpha, \beta>0, \alpha+\beta<|\Omega|$. Problems of this class have been encountered in the context of immissible fluids [8] and mixtures of micromagnetic materials [1]. The difficulty of such problems is the special structure of their constraints: a sequence of functions satisfying these constraints can have a limit which fails to satisfy the constraints.

Such minimization problems but with only one volume constraint have been studied by various authors, see e.g. [2]. Problems with two or more constraints have a very different nature than problems with only one volume constraint: in the case of one volume constraint, only additional boundary conditions or the design of the energy can induce transitions of the solution between different values. Two or more volume constraints,

\footnotetext{
Keywords and phrases. Volume constrained problems, numerical simulations, level set method, local minima.

* The second author is grateful for two invitations to the Université de Savoie that enabled this work.

1 Université de Savoie, France; Edouard.Oudet@univ-savoie.fr

2 University of Zürich, ISB, Switzerland; rieger@isb.uzh.ch
} 
on the other hand, force transitions of the solution by their very nature. Such problems have been studied starting from the fundamental work by Ambrosio et al. [4]. Their results have been generalized by various authors, compare e.g., $[10,11,15]$. It turned out that existence can only be guaranteed for functions $f$ satisfying quite specific conditions, and that there are easy examples of nonexistence, e.g. if $n=1, f\left(x, u, u^{\prime}\right)=\left|u^{\prime}\right|^{2}+|u|$ and $|\Omega|-\alpha-\beta$ sufficiently large [10]. Whereas the one dimensional case by now is relatively well understood (compare $[10,15]$ ), there are few sharp results on existence in the higher dimensional case [16]. There are in addition some results on local minimizers in the one-dimensional case [10], but there were so far no rigorous results in the higher dimensional case. By computing the shape derivative of the functional it is, however, possible to give a necessary condition for minimizers, as has been done in [4], Theorem 3.4:

Theorem 1.1. Let $u \in W^{1,2}(\Omega,[0,1])$ be a solution of (1.1). Assume that $S:=\partial\{u=0\} \cap \Omega$ is $C^{1}$, then $\frac{\partial u}{\partial n}$ is locally constant on $S$.

There is also very little known about explicit examples of minimizers in two dimensions, compare [4,15].

In this article we are introducing a numerical method for the approximation of local minimizers of (1.1). We apply this method to various examples and obtain a first picture of the shape of local and global minimizers for some simple domains in $\mathbb{R}^{2}$. Guided by the numerical results, we prove rigorously that even on the unit square solutions are not depending continuously on the parameter $\alpha$ and $\beta$ and illustrate this with numerical results. Moreover, we show that even on convex domains in $\mathbb{R}^{2}$ nontrivial local minimizers can exist.

\section{Numerical approximations}

\subsection{General approach and level-set methods}

We suppose in this section the existence of a solution of (1.1), i.e. that there exists a function $u \in H^{1}(\Omega)$ minimizing the problem (1.1). Our goal is to find a numerical method for the computation of this solution.

We will first explain our ideas in the simplest situation where $f(x, u(x), \nabla u(x))=|\nabla u(x)|^{2}$. In this situation existence of a solution for problem (1.1) has been already found in [4]. Our approach is based on the following fact: let $u^{*}$ be an optimal function for the problem, and denote

$$
\Omega_{a}=\left\{x \in \Omega, u^{*}(x)=a\right\}, \quad \Omega_{b}=\left\{x \in \Omega, u^{*}(x)=b\right\} .
$$

$\Omega_{a}$ and $\Omega_{b}$ are closed sets, since $u$ is Hölder continuous, for a proof see [11], Theorem 3.3. Then, it is possible to reconstruct $u^{*}$ by solving the elliptic boundary value problem:

$$
\left\{\begin{array}{cll}
\Delta u=0, & \text { in } & \Omega \backslash\left(\Omega_{a} \cup \Omega_{b}\right), \\
u=\alpha & \text { on } & \partial \Omega_{a} \\
u=\beta & \text { on } & \partial \Omega_{b}, \\
\frac{\partial u}{\partial n}=0 & \text { on } & \partial \Omega \backslash\left(\Omega_{a} \cup \Omega_{b}\right) .
\end{array}\right.
$$

The numerical approximation of an optimal function $u^{*}$ is hence reduced to an optimization problem for the two sets $\Omega_{a}$ and $\Omega_{b}$. Unfortunately, very few results are known concerning the optimal sets $\Omega_{a}$ and $\Omega_{b}$. In particular, it is not possible to restrict the optimization process to connected sets since disconnected sets can be optimal. We propose below an approach based on level set methods which makes it possible to generate also disconnected sets.

Before this, we recall briefly the standard tools of level set methods in a simplified context where only one single shape is unknown (see for instance [12] for numerical details closely related to our approach). We explain later how to deal with more than one unknown shape.

Let $\Omega$ be a subset of $\mathbb{R}^{2}$, we consider an optimization problem where we want to find an optimal set $\mathcal{O} \subset \Omega$ for a given functional. The main idea of the method is to parameterize $\mathcal{O}$ by a function $\Phi$, the so-called 
level set function, that satisfies

$$
\begin{cases}\Phi(x)<0 & \text { if } x \in \mathcal{O} \\ \Phi(x)>0 & \text { if } x \in \Omega \backslash \overline{\mathcal{O}} \\ \Phi(x)=0 & \text { if } x \in \partial \mathcal{O}\end{cases}
$$

For numerical convenience which will be explain below, the level set function $\Phi$ is always defined on a Cartesian grid defined on a square containing the set $\Omega$.

As suggested in [13], such a function will be initialized with the signed-distance which is given by

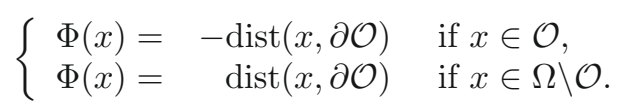

We remark that the constructed distance is generally not easy to compute. In our case, for the Cartesian mesh on $\Omega$, deduced by the Cartesian grid where $\Phi$ is defined, we choose an approximate signed-distance function which is constant on each triangle of the mesh. Its value in the triangle $T$ is computed by evaluating the distance between the center of mass of $T$ and the center of mass of the closest triangle lying on the boundary of the initial shape.

Once $\Phi$ is defined, we can let its level set at 0 (i.e. $\partial \mathcal{O}$ ) fluctuate with time under the vector field $v n$ (where $v$ is a real-valued function and $n$ is the normal vector on $\partial \mathcal{O})$. In other words, if $x(t)$ describes the evolution of a point on $\partial \mathcal{O}$ under such a transformation, it has to satisfy

$$
\Phi(t, x(t))=0
$$

for all $t$. Differentiating this expression, we obtain

$$
\frac{\partial \Phi}{\partial t}(t, x(t))+v(x(t)) n(x(t)) \cdot \nabla_{x} \Phi(t, x(t))=0
$$

Now the normal to a level set in a non-stationary point is given by

$$
n(x(t))=\frac{\nabla_{x} \Phi}{\left|\nabla_{x} \Phi\right|}(t, x(t)) .
$$

Hence, using (2.2), we derive

$$
\frac{\partial \Phi}{\partial t}(t, x(t))+v(x(t))\left|\nabla_{x} \Phi\right|(t, x(t))=0 .
$$

In order to compute the evolution of $\Phi$, we thus have to solve a Hamilton-Jacobi equation. We remark that the computation we have presented only concerns the level set 0 , but since in practice the vector field $v n$ has a natural extension on $\Omega$, we solve the equation (2.3) in the whole set $\Omega$.

We want to find a good velocity field $v n$ for the shape optimization problem under investigation. Therefore we follow an approach which has been first introduced in [3] and choose $v n$ as the vector field obtained by boundary variations. Let $\mathcal{O} \subset \Omega$ be a connected set with $C^{2}$-boundary and $u$ a solution of the problem

$$
\left\{\begin{array}{cll}
\Delta u=0, & \text { in } & \Omega \backslash \mathcal{O} \\
u=\alpha & \text { on } & \partial \mathcal{O} \\
\frac{\partial u}{\partial n}=0 & \text { on } & \partial \Omega \backslash \mathcal{O} .
\end{array}\right.
$$

It is well known in shape optimization (see for instance $[6,9,17]$ ) that the shape derivative of the energy of $u$ in the direction of a vector field $V$ localized around $\partial \mathcal{O}$ is given by Hadamard's formula

$$
\frac{\mathrm{d} E}{\mathrm{~d} V}=-\int_{\partial \mathcal{O}}\left(\frac{\partial u}{\partial n}\right)^{2} V n \mathrm{~d} \sigma .
$$


This computation suggests that the steepest descent direction is given by the normal vector field

$$
-\left(\frac{\partial u}{\partial n}\right)^{2} n
$$

Moreover, since $u$ is by definition constant along $\partial \mathcal{O}$ this vector field has a natural extension to the domain $\Omega$ using the relation:

$$
n= \pm \frac{\nabla \Phi}{|\nabla \Phi|}
$$

In order to avoid the computation of a new mesh at each iteration, we compute an approximation of the solution of (2.4) via a penalization method introduced in [14].

\subsection{A multi-level set method}

As explained before, the numerical approximation of (1.1) can be reduced to the approximation of the two sets $\{x \in \Omega, u(x)=a\}$ and $\{x \in \Omega, u(x)=b\}$. In that case, two shapes are unknown and we propose to parameterize those sets with two different level set functions, namely $\Phi_{a}$ and $\Phi_{b}$. At each step of the algorithm the two sets evolve under the local vector field given by the shape derivative. The only point that we have to worry about is the possibility of crossing of those level sets. Several approaches have already been investigated for dealing with this kind of difficulty. The most standard way to avoid the crossing of the level sets is to add a penalization term like

$$
\int_{\Omega}\left(H\left(\Phi_{a}(x)\right)+H\left(\Phi_{b}(x)\right)-1\right)^{+} \mathrm{d} x=0
$$

to the functional, where $H(y)$ is equal to 1 for $y<0$ and equal to 0 otherwise and $(y)^{+}$stands for the positive part of $y$. Although we are not able to prove that the crossing of level sets will never happen during the optimization, we did not need to implement the previous method, since in our simulations, we never observed a crossing of level sets. This fact is probably a result of the fact that such crossing (or even touching) of the level sets cannot occur in the limit, i.e. for minimizers of (1.1) as the following theorem states:

Theorem 2.1. Let $u$ be a global minimizer of (1.1). Then $\operatorname{dist}(\{u=a\},\{u=b\})>0$.

Proof. This is an immediate consequence of a regularity result by Mosconi and Tilli [11] that ensures that $u$ is Hölder continuous.

Of course, this idea can be extended to arbitrary numbers of level sets.

We now compute the solution of the above Hamilton-Jacobi equation. Our description will be limited to a simple algorithm reported in [13] designed to approach the weak viscosity solution of the Hamilton-Jacobi equation problem. Let us consider the first order Cauchy system:

$$
\left\{\begin{array}{l}
\frac{\partial \Phi}{\partial t}(t, x)-F(x)|\nabla \Phi(t, x)|=0 \quad \text { in } \mathbb{R}_{+} \times D \\
\Phi(0, x)=u_{0}(x) \text { in } D
\end{array}\right.
$$

where $D$ is a bounded rectangle of $\mathbb{R}^{2}$ and $u_{0}$ and $F$ are given functions. From now on we shall use the classical notations for finite difference schemes on regular meshes of points indexed by $i, j$. Starting from $\Phi(0, x)=u_{0}(x)$, then the evolution of $\Phi$ after one time step $\Delta t$ is given by

$$
\Phi_{i j}^{n+1}=\Phi_{i j}^{n}-\Delta t\left(\max \left(F_{i j}, 0\right) \nabla^{+} \Phi+\min \left(F_{i j}, 0\right) \nabla^{-} \Phi\right),
$$

where

and

$$
\nabla^{+} \Phi=\left[\max \left(D_{i j}^{-x} \Phi, 0\right)^{2}+\min \left(D_{i j}^{+x} \Phi, 0\right)^{2}+\max \left(D_{i j}^{-y} \Phi, 0\right)^{2}+\min \left(D_{i j}^{+y} \Phi, 0\right)^{2}\right]^{1 / 2}
$$

$$
\nabla^{-} \Phi=\left[\max \left(D_{i j}^{+x} \Phi, 0\right)^{2}+\min \left(D_{i j}^{-x} \Phi, 0\right)^{2}+\max \left(D_{i j}^{+y} \Phi, 0\right)^{2}+\min \left(D_{i j}^{-y} \Phi, 0\right)^{2}\right]^{1 / 2}
$$


with

$$
D_{i j}^{+x} \Phi=\frac{\Phi_{i+1, j}-\Phi_{i, j}}{\Delta x}
$$

for a space step equal to $\Delta x$. The quantities $D_{i j}^{-x} \Phi, D_{i j}^{+y} \Phi$ and $D_{i j}^{-y} \Phi$ are easily deduced. Finally, to define completely our problem, we add the boundary condition

$$
\frac{\partial \nabla \Phi(t, x)}{\partial n}=0 \text { on } \partial D
$$

The volume of the level set function $\Phi_{a}$ at the discrete level is by definition the volume of all the elements of the mesh where $\Phi_{a}$ is less or equal than zero. In order to preserve this volume equal to $\alpha$ along the iterations, we use the Lagrange multiplier technique reported in [12]. According to the derivative computed in (2.3), the level set function $\Phi_{a}$ satisfies the Hamilton-Jacobi equation

$$
\frac{\partial \Phi_{a}}{\partial t}(t, x)-\left(-|\nabla u|^{2}(t, x)+\mu\right)\left|\nabla \Phi_{a}(t, x)\right|=0 \text { in } \mathbb{R}_{+} \times D
$$

where $u(t,$.$) is the solution of the system (2.1)$ associated to $\Phi_{a}(t,$.$) and \Phi_{b}(t,$.$) . As suggested by Osher and$ Santosa [12], at each iteration we adapt the Lagrange multiplier $\mu$ to preserve the volume constraint. The same projection method is of course reproduced for the level set function $\Phi_{b}$, in case of two volume constraints.

It is now possible to describe all the steps of our algorithm:

1. Initialization of $\Phi_{a}$ and $\Phi_{b}$ by the signed distance on a Cartesian grid containing $\Omega$.

2. Computation of the velocity field by a penalization method introduced in [14] on the fixed triangular mesh deduced from the Cartesian grid. Checking of an exit criterion.

3. Propagation of the level sets solving the Hamilton-Jacobi equations (2.5) preserving the volume constraints.

4. Evaluation of the cost function. If the cost decreases then go to step 5. Otherwise divide the time step by 1.5 and go to step 3 .

5. Redefinition of $\Phi_{a}$ and $\Phi_{b}$.

6. Eventually, reinitialization of $\Phi_{a}$ and $\Phi_{b}$ with the signed distance. Back to step 2.

For more details on the computation of the solution of the state equation associated to $\Phi_{a}$ and $\Phi_{b}$ (in the context of one level set constraint) see [3] or [14].

\subsection{Examples}

We present the result of our optimization process in the next figures. We first study the problem (1.1) with $\Omega$ a disc of radius $0.45, \alpha=\beta=0.15^{2} \pi, a=0$ and $b=1$. We obtain the same optimal shape with different initial guesses presented in Figures 1 and 2. The algorithm which has been presented in the case of two constraints can easily be adapted to a situation with more constraints. We present in Figure 3 our results for a problem with three constraints of equal volume $0.15^{2} / 2$.

\section{SOlution PROPERTIES}

\subsection{Illustration of nonexistence results}

It had been pointed out in $[10,11]$ that problems of the type (1.1) in general do not have solutions. However, the relaxed problem

$$
\begin{array}{r}
\text { Minimize } E(u):=\int_{\Omega} f(x, u(x), \nabla u(x)) \mathrm{d} x, \\
|\{x \in \Omega, u(x)=a\}| \geq \alpha, \\
|\{x \in \Omega, u(x)=b\}| \geq \beta,
\end{array}
$$



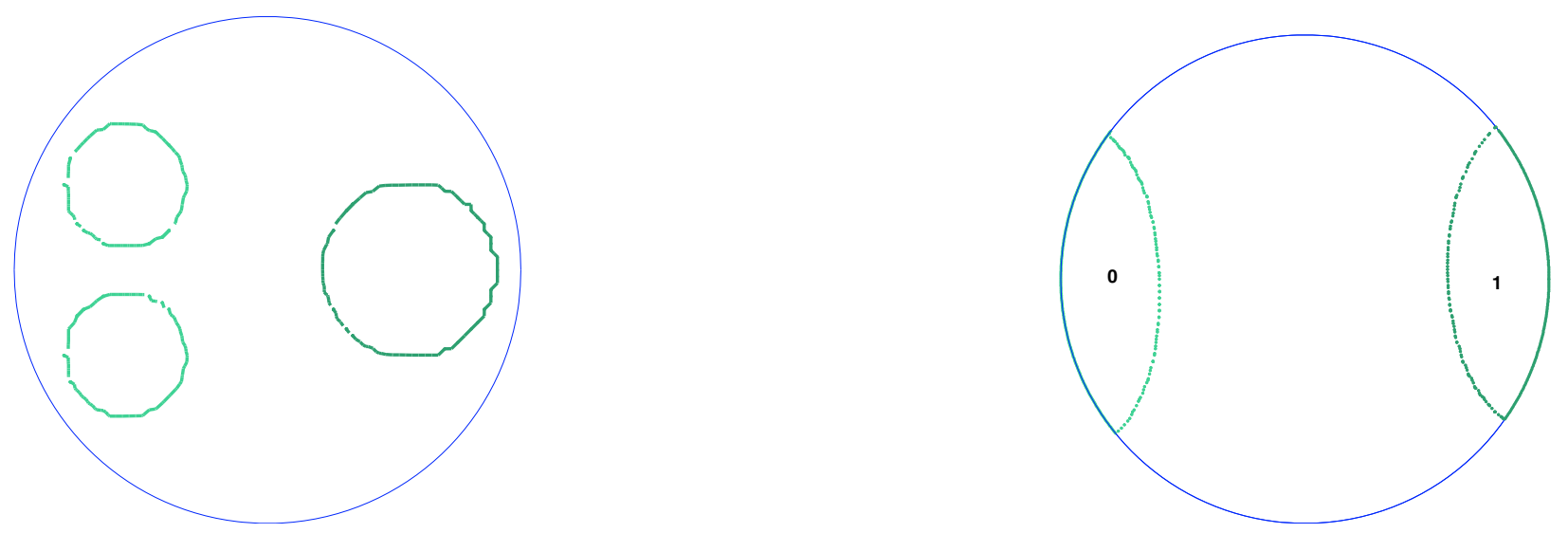

FIGURE 1. Initial and optimized level sets for a problem with two constraints.

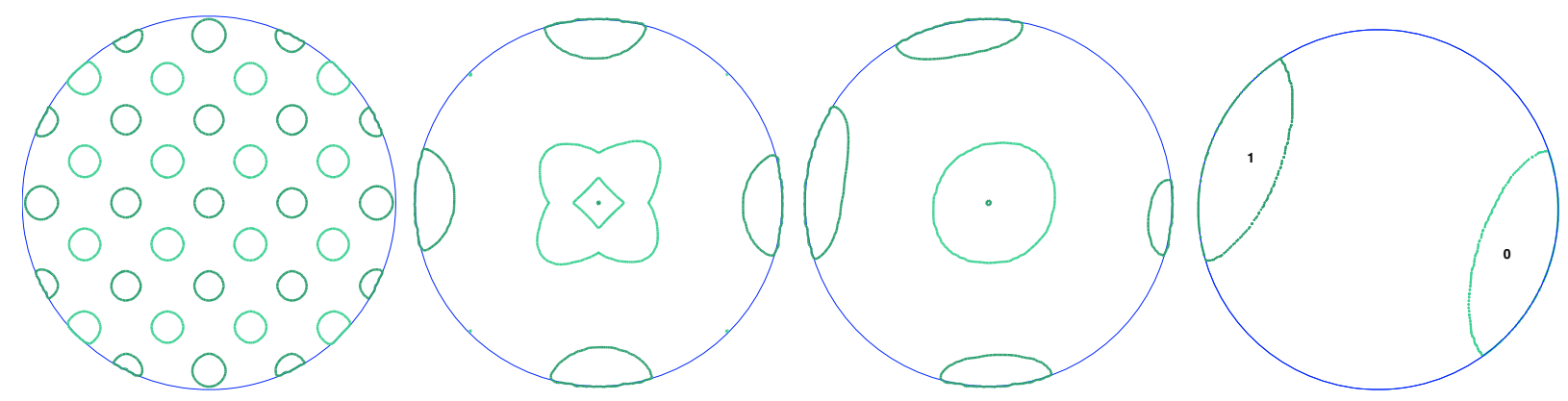

Figure 2. Evolution of the level sets for a problem with two constraints (the same as the ones of Fig. 1).

admits a solutions whenever $f$ satisfies some standard convexity and growth conditions [4]. Our previous numerical computations solve (3.1), and in the case of $f(x, u, \nabla u)=|\nabla u|^{2}$ it has been proved already in [4] that any solution of (3.1) also solves (1.1).

In this subsection we want to consider a situation where existence of a solution for (1.1) fails. To this aim we choose $f(x, u, \nabla u)=|\nabla u|^{2}+|u|$ and try to compute numerically a solution of the ill-posed problem (3.1) for $a=0, b=1$ and $\alpha=\beta=\pi(0.15)^{2}$ on the unit disk $\Omega$. As we can observe in Figure 4, the resulting level set of the constraint corresponding to $a=0$ is strictly larger than the one which is prescribed. Actually, the area of that level set is approximatively equal to $0.0872>\pi(0.15)^{2}$. In that sense, our numerical simulation illustrates the fact that non existence can occur for problem (1.1).

\subsection{Discontinuous parameter dependence}

If $u^{\alpha, \beta}$ denotes the solution to a volume constrained problem of the type (1.1) then it is a natural question whether $u^{\alpha, \beta}$ depends (in an appropriate sense) continuously on $\alpha$ and $\beta$. It turns out that this is in general not the case, in fact we have the following result:

Theorem 3.1. If we set $f(u, \nabla u)=|\nabla u|^{2}$ and $\Omega=(0,1)^{2}$ then the minimizers $u^{\alpha, \beta}$ of the problem (1.1) do not depend continuously on $\alpha$ and $\beta$, more precisely: there is an $\varepsilon>0$ such that $\alpha \mapsto u^{\alpha, 1-\alpha-\varepsilon}$ is not continuous in $\alpha$ with respect to the $L^{1}$-norm.

To prove this result we use the $\Gamma$-limit of the problem (1.1). We briefly recall the definition of $\Gamma$-convergence and refer the reader for any details to the books of Braides [5] and Dal Maso [7]: 

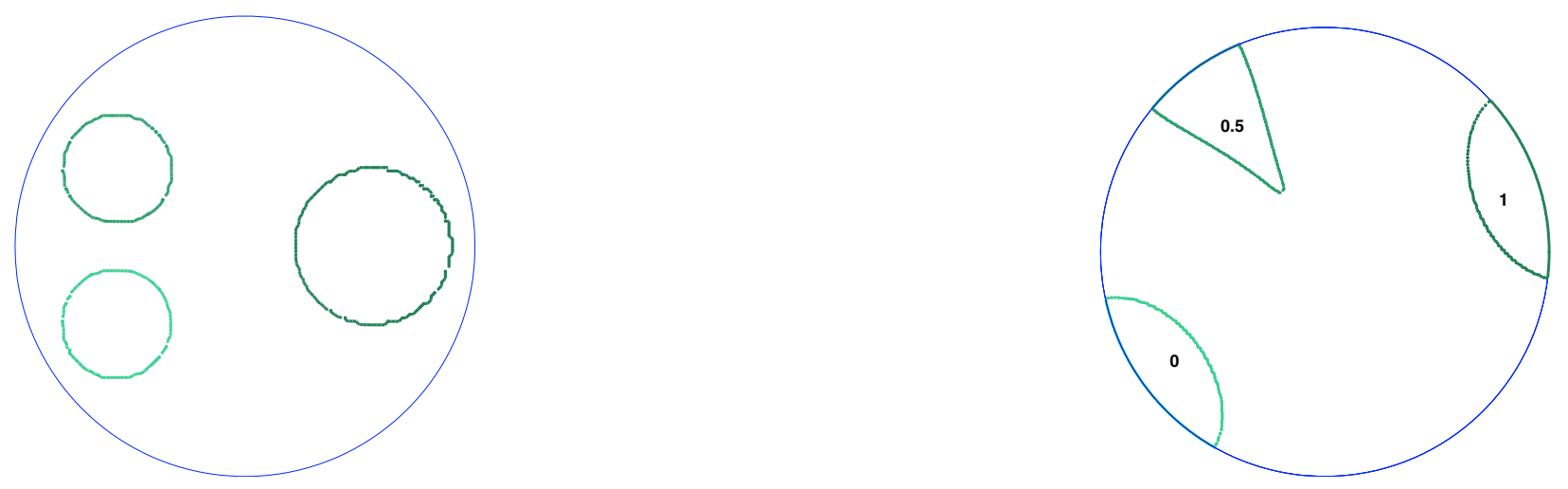

Figure 3. Initial and optimized level sets for a problem with three constraints.

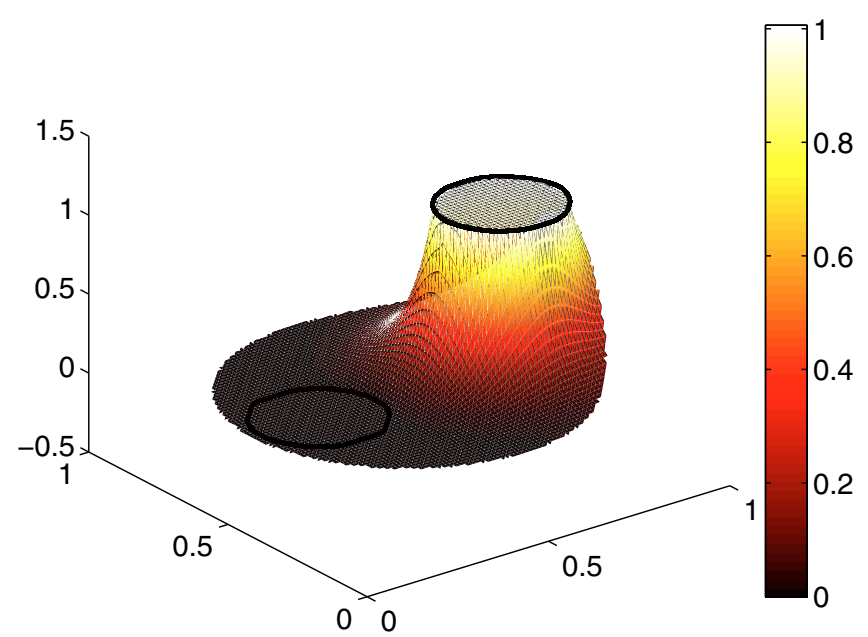

Figure 4. Computed minimizer $u$ of a relaxed problem (3.1) which does not satisfy the constraints of the exact problem (1.1), since its zero level set is too big. This illustrates the nonexistence of solutions for (1.1) in the two-dimensional case (see text for details).

Definition 3.2 ( $\Gamma$-convergence). Let $F_{n}$ be a sequence of functionals on a Banach space $X$. Then we say that $F_{n}$ is $\Gamma$-converging in $X$ to the functional $F$ and denote $X-\Gamma-\lim F_{n}=F\left(\right.$ or $F_{n} \stackrel{\Gamma}{\rightarrow} F$ ) if

(i) For every $u \in X$ and for all $u_{n} \rightarrow u$ in $X$ we have

$$
\liminf _{n \rightarrow \infty} F_{n}\left(u_{n}\right) \geq F(u)
$$

(ii) For every $u \in X$ there exists a sequence $u_{n} \subset X$ such that $u_{n} \rightarrow u$ and

$$
\limsup _{n \rightarrow \infty} F_{n}\left(u_{n}\right) \leq F(u)
$$

Inequality (3.2) is called $\Gamma$-liminf inequality and (3.3) is called $\Gamma$-limsup inequality. Such a $\Gamma$-limit has been derived for the case $\alpha+\beta \rightarrow 1$ and $f(u, \nabla u)=|\nabla u|^{2}$ in [4]. A generalization can be found in [16]. Let $\Omega \subset \mathbb{R}^{N}$ 

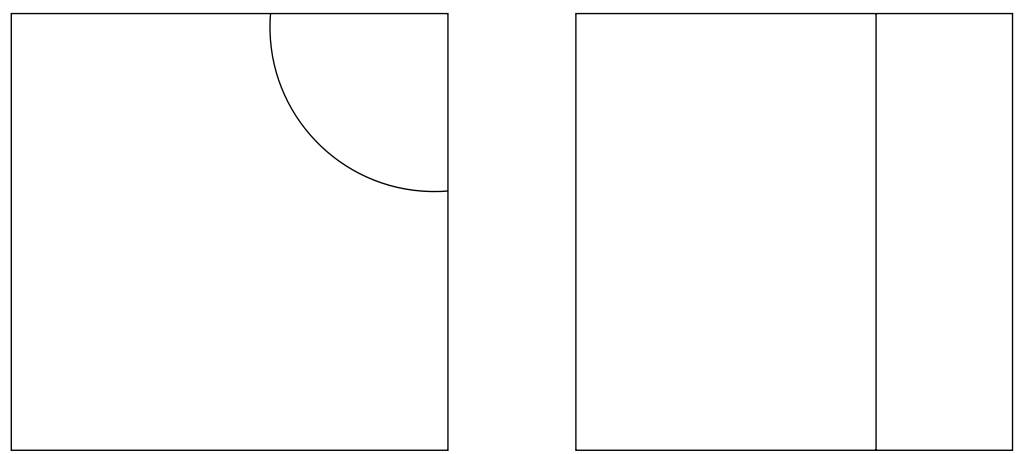

Figure 5. Type I and type II solutions.

be an bounded open set. For fixed $\alpha, \beta \in(0,|\Omega|)$, we define the following functional

$$
F_{\alpha, \beta}:= \begin{cases}\gamma \int_{\Omega}|\nabla u|^{2} \mathrm{~d} x & \text { if } u \in \mathcal{A}_{\alpha, \beta} \\ +\infty & \text { elsewhere in } L^{1}(\Omega)\end{cases}
$$

where $\gamma:=|\Omega|-(\alpha+\beta)$ and

$$
\mathcal{A}_{\alpha, \beta}:=\left\{u \in H^{1}(\Omega):|\{u=0\}|=\alpha \text { and }|\{u=1\}|=\beta\right\} .
$$

Then we can state the theorem from [4] as follows:

Theorem 3.3. Let $\bar{\alpha} \in(0,|\Omega|)$. Then

$$
\Gamma\left(L^{1}\right)-\lim _{\substack{\alpha \rightarrow \bar{\alpha} \\ \beta \rightarrow|\Omega|-\bar{\alpha}}} F_{\alpha, \beta}=G_{\bar{\alpha}}
$$

with $G_{\bar{\alpha}}$ given by

$$
G_{\bar{\alpha}}:= \begin{cases}\mathscr{H}^{1}(\{u=0\})^{2} & \text { if } u \in B V(\Omega,\{0,1\}) \text { and }|\{u=0\}|=\bar{\alpha} \\ +\infty & \text { elsewhere in } L^{1}(\Omega)\end{cases}
$$

This limit problem is much more accessible to analytical investigations. In particular we can set $A:=\{u=0\}$ and $B:=\{u=1\})$ and then the minimizers of $G_{\alpha}$ correspond to minimizers of the Dido's problem [18]: minimize $\mathscr{H}^{1}(\Gamma)$ such that $\Gamma$ separates $\Omega$ in open sets $A$ and $B$ with $|A|=\alpha$ and $|B|=|\Omega|-\alpha$. The solutions of this problem can be explicitly computed. In the following lemma we summarize the situation on the unit square:

Lemma 3.4. Let $\Omega=(0,1)^{2}, \alpha>0$, then there exists a set $\Gamma \subset \Omega$ minimizing $\mathscr{H}^{1}(\Gamma)$ among all sets with the property that there exist disjoint open sets $A, B \subset \Omega \backslash \Gamma$ with $|A|=\alpha,|B|=1-\alpha$ and $\Omega=A \cup B \cup \Gamma$.

(i) If $\alpha<1 / \pi$ or $\alpha>1-1 / \pi$ then $\Gamma$ is the segment of a circle with center in one of the corner points of $\Omega$. (Type I solution, see Fig. 5.)

(ii) If $1 / \pi<\alpha<1-1 / \pi$ then $\Gamma$ is a straight line parallel to a side of $\Omega$. (Type II solution, see Fig. 5.)

(iii) If $\alpha=1 / \pi$ or $\alpha=1-1 / \pi$ then $\Gamma$ is either a circle segment or a straight line. 
$\Omega$
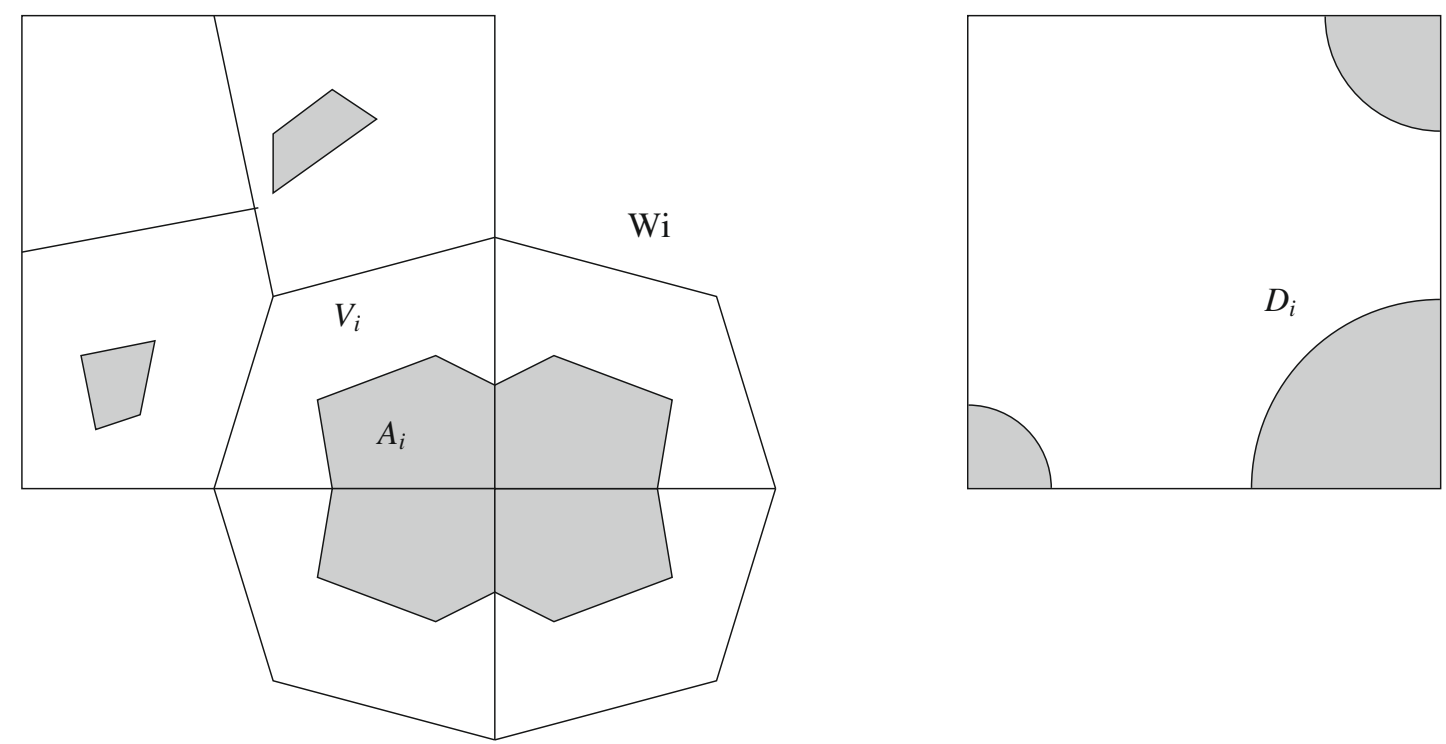

Figure 6. The construction for the proof of Lemma 3.4.

This lemma seems to be folklore, but for the reader's convenience we give a proof using the isoperimetric inequality:

Proof. By symmetry we can assume that $\Gamma$ is a solution of the problem for $\alpha \in(0,1 / 2]$, moreover we assume first that $\ell:=\mathscr{H}^{1}(\Gamma)<1$. Denote the four corner points in the square $\Omega$ by $Q_{i}$ and the sides by $S_{i}$. Since $\ell<1$ the set projection $\pi_{i}$ of $\Gamma$ onto $S_{i}$ satisfies $\pi_{i}(\Gamma) \neq S_{i}$. Let $x \in S_{1} \backslash \pi_{1}(\Gamma)$ and $y \in S_{2} \backslash \pi_{2}(\Gamma)$. Then the cross-shaped set $\left\{\left(x_{1}, x_{2}\right) \in \Omega \mid x_{1}=x\right.$ or $\left.y_{1}=y\right\}$ does not intersect with $A$, therefore we can decompose $\Omega$ along this cross into four disjoint connected open sets $V_{1}, \ldots, V_{4}$ such that $\bigcup_{i} \bar{V}_{i}=\bar{\Omega}$ and each $\bar{V}_{i}$ contains the corner point $Q_{i}$ and none of the other corner points. We observe that since $V_{i}$ open, $\partial V_{i} \cap A \subset \partial \Omega$. We can now mirror $V_{i}$ and $A \cap V_{i}$ three times along the adjacent sides of the square $\Omega$ (see Fig. 6) to obtain a larger set $A_{i} \subset \mathbb{R}^{2}$. Since $\partial A \cap \partial V_{i}$ was a subset of the mirror axis, we can now neglect the boundary and apply the isoperimetric inequality on the sets $A_{i}$, hence proving that they minimize their boundary length (under fixed volume) when they are discs. We can center these disks without loss of generality on $Q_{i}$ and denote them by $D_{i}$ and $D:=\bigcup_{i} D_{i}$. Due to the minimality property of the boundary length, we have $\ell=\mathscr{H}^{1}(\Gamma) \geq \frac{1}{4} \sum_{i} \mathscr{H}^{1}\left(\partial D_{i}\right)$. Since $\ell<1$, the disks $D_{i}$ must be disjoint. (Otherwise the sum of two of their radii $r_{i}$ would have to exceed the distance between two corner points, i.e. 1 , but that would imply $1>\ell \geq\left(r_{1}+r_{2}\right) 2 \pi / 4>\pi / 2$.) Since the disks are disjoint, we have $|D|=\sum_{i}\left|D_{i}\right|=|A|$. For the boundary length we have seen that $\mathscr{H}^{1}(\Gamma) \geq \frac{1}{4} \sum_{i} \mathscr{H}^{1}\left(\partial D_{i}\right)$ with equality if and only if $\Gamma$ consists of at most four arcs with centers in $Q_{i}$. It is now easy to check that the optimal configuration among these sets is given by exactly one arc with center in some $Q_{i}$. Since our initial assumption $\ell<1$ is feasible if $\alpha<1 / \pi$, we have proved the first point of the theorem.

The last two points of the theorem follow easily: we know that in both cases there exists a $\Gamma$ with $\mathscr{H}^{1}(\Gamma)=1$. Suppose we could do better, then $\Gamma$ would satisfy $\mathscr{H}^{1}(\Gamma)<1$ and we could apply the argument above, proving that $\Gamma$ must be an arc with center in some $Q_{i}$. Such an arc, however, would have a length larger than 1 (or in the case $\alpha=1 / \pi$ at least not less) which contradicts the assumption.

Proof of Theorem 3.1. Assume that for all $\varepsilon>0$ the function $h_{\varepsilon}(\alpha):=u^{\alpha, 1-\alpha-\varepsilon}$ is continuous in the $L^{1}$-norm. We know by the $\Gamma$-convergence that $u^{\alpha, 1-\alpha-\varepsilon} \rightarrow u^{\alpha}$ in $L^{1}$ where $u^{\alpha}$ denotes the minimizer of the $\Gamma$-limit problem. Hence, for $\alpha<1 / \pi$ the functions $h_{\varepsilon}(\alpha)$ converge to a limit function $h(\alpha)$ of the type I as $\varepsilon \rightarrow 0$ 

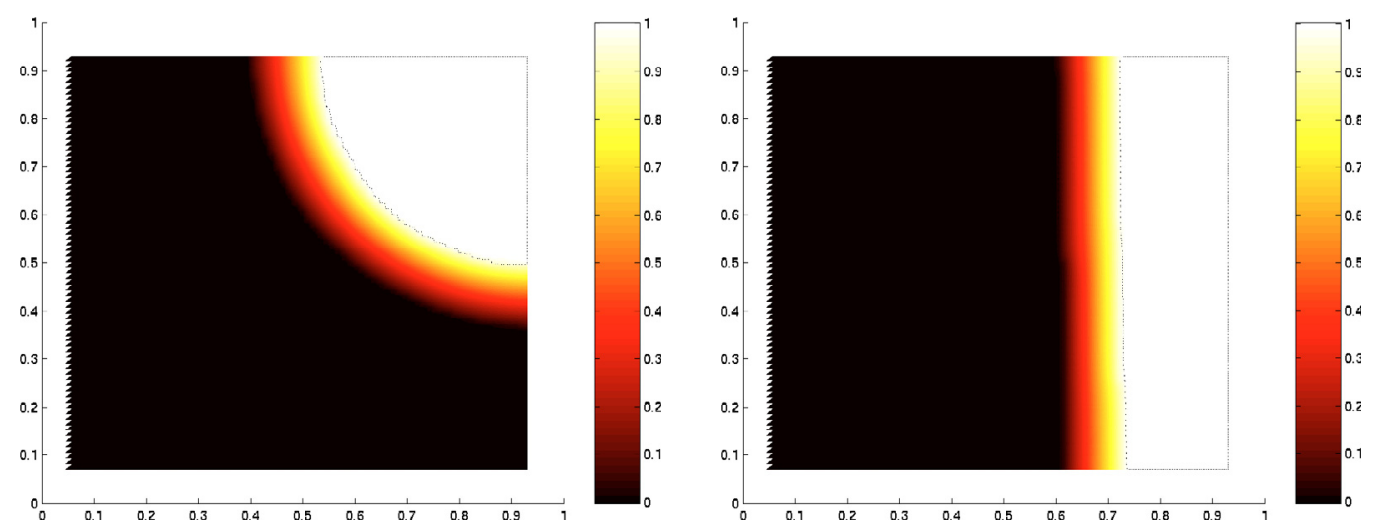

FiguRE 7. Global minimizers for the parameters $\alpha=0.55, \beta=0.15$ (left) and $\alpha=0.5, \beta=0.2$ (right) on a square with side length 0.9 . Although the parameters are very close, the solutions are not.

(see Fig. 5), for $1 / \pi \alpha<1-1 / \pi$, however, the functions $h_{\varepsilon}(\alpha)$ converge to a function of the type II (see Fig. 5). For $\alpha=1 / \pi$ we denote the two possible solutions of the limit problem by $u^{I}$ and $u^{I I}$. The $L^{1}$-distance between $u^{I}$ and $u^{I I}$ is larger than 0.6 (as a small computation shows). We do not necessarily have uniform convergence of $h_{\varepsilon}$ as $\varepsilon \rightarrow 0$, hence we need the following construction:

Let us fix $\alpha^{1}, \alpha^{2}$ such that $\alpha^{1}<1 / \pi<\alpha^{2}$ and

$$
\left\|h\left(\alpha^{1}\right)-u^{I}\right\|,\left\|h\left(\alpha^{2}\right)-u^{I I}\right\|<1 / 100
$$

(We can ensure this by choosing $\alpha^{1}$ and $\alpha^{2}$ close to $1 / \pi$ since the minimizers of the limit problem are continuous outside $1 / \pi$.)

Next, we choose sequences $\alpha_{n}^{1}, \alpha_{n}^{2}$ and $\varepsilon_{n}$, such that $\varepsilon_{n}<1 / n, \alpha_{n}^{1} \rightarrow \alpha^{1}, \alpha_{n}^{2} \rightarrow \alpha^{2}$ and $\left\|h_{\varepsilon_{n}}\left(\alpha_{n}^{1}\right)-h\left(\alpha^{1}\right)\right\|<$ $1 / n,\left\|h_{\varepsilon_{n}}\left(\alpha_{n}^{2}\right)-h\left(\alpha^{2}\right)\right\|<1 / n$. (By the $\Gamma$-convergence we know that minimizers of the volume constraint problem converge for $\varepsilon \rightarrow 0$ to minimizers of the limit problem, hence we can find such sequences.)

Now we choose a sequence of $\alpha_{n}^{0}$ that lies in between $\alpha_{n}^{1}$ and $\alpha_{n}^{2}$ and prove that the corresponding solutions of the volume constrained problem cannot converge to a solution of the limit problem:

Let $\alpha_{n}^{0}$ satisfy $\alpha_{n}^{1}<\alpha_{n}^{0}<\alpha_{n}^{2}$. Using the (supposed) continuity of $h$ we can apply the intermediate value theorem to find such an $\alpha_{n}^{0}$ such that $\left\|h_{\varepsilon_{n}}\left(\alpha_{n}^{0}\right)-h_{\varepsilon_{n}}\left(\alpha_{n}^{1}\right)\right\|>1 / 10$ and $\left\|h_{\varepsilon_{n}}\left(\alpha_{n}^{0}\right)-h_{\varepsilon_{n}}\left(\alpha_{n}^{2}\right)\right\|>1 / 10$. Since the sequence $\alpha_{n}^{0}$ is uniformly bounded, we can select a converging subsequence and, using the $\Gamma$-converge, its limit $\alpha^{0}$ satisfies $\left\|h\left(\alpha^{0}\right)-h\left(\alpha^{1}\right)\right\| \geq 1 / 10$ and $\left\|h\left(\alpha^{0}\right)-h\left(\alpha^{2}\right)\right\| \geq 1 / 10$.

Using this together with (3.5) and $\left\|u^{I}-u^{I I}\right\|>0.6$ leads to a contradiction. Hence at least for sufficiently small $\varepsilon>0$ the function $h_{\varepsilon}$ cannot be continuous.

We illustrate this behavior with numerical computations (Fig. 7) using the algorithm introduced in Section 2.

\subsection{Existence of local minimizers}

Our algorithm searches for minimizers which are not necessarily global minimizers. In one dimension it was possible to characterize local minimizers completely with analytical methods [10]. However, on convex domains of dimension $n \geq 2$ these methods do not work and it had been conjectured that in fact every minimizer is global. It is relatively simple to see examples of local minimizers in nonconvex domains (compare Fig. 8 for a numerical computation). However, our computation hinted that also on the square there can be genuinely local minimizers, compare Figure 9.

In the following we present a proof of the existence of genuinely local minimizers on a square. 

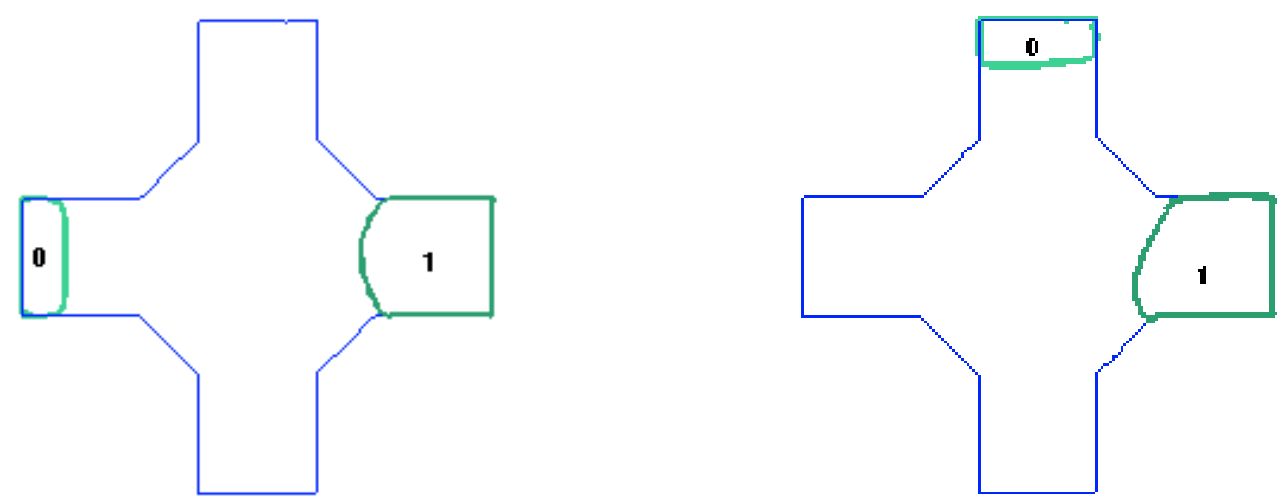

Figure 8. Global (left) and local (right) minimizer on a nonconvex domain.
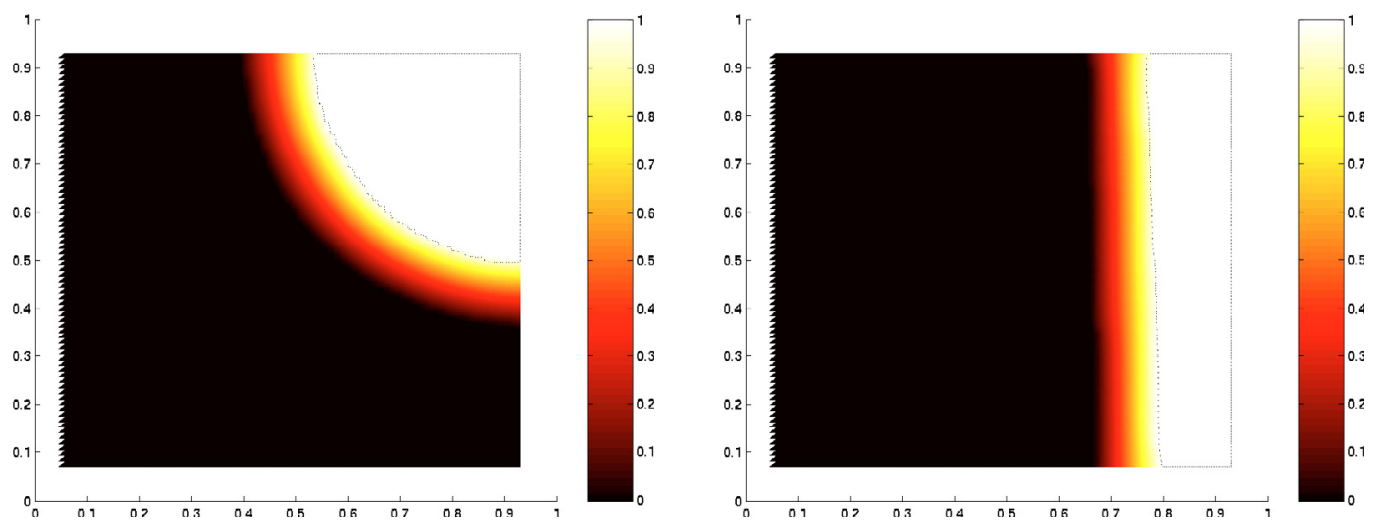

Figure 9. Global (left) and local minimizer of the same problem as shown in the left side of Figure 7. This example demonstrates that there are genuinely local minimizers on a convex domain, in this case a square.

Theorem 3.5 (Existence of local minimizer). There are convex domains $\Omega \subset \mathbb{R}^{2}$ such that the volumeconstrained minimization problem (1.1) with $f(x, u, \nabla u)=|\nabla u|^{2}$ admits (for appropriate parameters) local minimizers (with respect to the $L^{\infty}$-distance) which are not global.

Proof. Let $\Omega$ be the unit square $(0,1) \times(0,1)$. For simplicity, $a=0$ and $b=1$. We choose $\alpha<\frac{1}{\pi}$ and $\beta=1-\alpha-\gamma$ where $\gamma>0$ is chosen small enough such that

$$
\gamma<\frac{\alpha}{2}
$$

We define our candidate $v$ for a local minimizer by a one-dimensional piecewise affine construction:

$$
v(x, y):=\left\{\begin{array}{cl}
1, & x<\beta \\
\frac{1-\alpha-x}{\gamma}, & \beta \leq x<1-\alpha \\
0, & 1-\alpha \leq x .
\end{array}\right.
$$

We compute the energy of $v$ as

$$
\int_{\Omega}|\nabla v|^{2}=\int_{0}^{\gamma}\left|\frac{\mathrm{d}}{\mathrm{d} x} \frac{x}{\gamma}\right|^{2}=\frac{1}{\gamma}
$$


For $\gamma \rightarrow 0$, the function $v$ converges in $L^{1}$ to a local minimizer of the $\Gamma$-limit functional which is not a global minimizer, compare Lemma 3.4. Therefore, for $\gamma>0$ sufficiently small, $v$ cannot be a global minimizer. It is therefore sufficient to prove that it is a local minimizer.

Let us suppose that there is another function $w$ in the neighborhood of $v$ with a smaller energy, more precisely suppose

$$
\|w-v\|_{L^{\infty}}<1 / 3
$$

and $\int_{\Omega}|\nabla w|^{2}<\int_{\Omega}|\nabla v|^{2}-\varepsilon$ for some $\varepsilon>0$. Assume furthermore that $w$ satisfies the same volume constraint as $v$. A priori, $w$ does not need to be continuous. For the further construction it is, however, pivotal to work with a continuous function. Therefore we show that it is possible to construct a continuous function $\tilde{w}$ with the same properties:

We observe first, that $w$ cannot have a "jump from zero to one", i.e. there cannot be a point $x \in \Omega$ such that there are sequences $x_{n}$ and $x_{n}^{\prime}$, both converging to $x$ with $w\left(x_{n}\right) \rightarrow 0$ and $w\left(x_{n}^{\prime}\right) \rightarrow 1$ : if such a point existed, then (thanks to the continuity of $v$ ) we have $\left|w\left(x_{n}\right)-v\left(x_{n}\right)+v\left(x_{n}^{\prime}\right)-w\left(x_{n}^{\prime}\right)\right| \rightarrow 1$. On the other hand, using (3.8), we have $\left|w\left(x_{n}\right)-v\left(x_{n}\right)\right|<1 / 3$ and $\left|w\left(x_{n}^{\prime}\right)-v\left(x_{n}^{\prime}\right)\right|<1 / 3$. Together with the triangle inequality, this leads to a contradiction.

We denote $\Omega_{0}:=\{x \in \Omega \mid w(x)=0\}$ and $\Omega_{1}:=\{x \in \Omega \mid w(x)=1\}$. Since there is no jump from zero to one, we have $\bar{\Omega}_{0} \cap \bar{\Omega}_{1}=\emptyset$ and we can therefore define

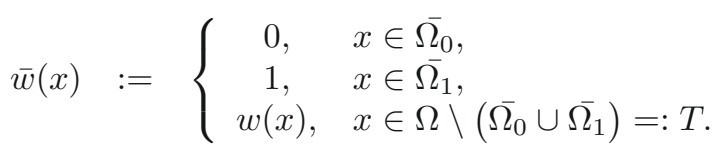

The set $T$ is open by construction. For each $x \in \partial T \backslash \partial \Omega$ there is either a sequence $x_{n} \rightarrow x$ such that $w\left(x_{n}\right) \rightarrow 0$ or a sequence $x_{n}^{\prime} \rightarrow x$ such that $w\left(x_{n}^{\prime}\right) \rightarrow 1$. Denote the corresponding sets of boundary points by $D_{0}$ and $D_{1}$, then $D_{0}$ and $D_{1}$ form a disjoint union of $\partial T \backslash \partial \Omega$. Moreover, given that $w$ has no jump from zero to one, $D_{0}$ and $D_{1}$ must be apart from each other, i.e. $\bar{D}_{0} \cap \bar{D}_{1}=\emptyset$. In other words, on $\partial T \backslash \partial \Omega, \bar{w}$ is locally constant.

The function $\bar{w}$ is by construction in $H^{1}(T)$, where $T$ is open. Thus we can approximate $\bar{w}$ on $T$ by continuous functions in the $H^{1}$-norm, where we respect the boundary conditions on $\partial T \backslash \partial \Omega$. Let $w_{n}$ be such an approximating sequence, then for $n$ large enough, $\left\|w_{n}-\bar{w}\right\|_{H^{1}(T)}<\varepsilon / 2$.

We can now define $\tilde{w}$ by

$$
\tilde{w}(x):=\left\{\begin{array}{cl}
0, & x \in \overline{\Omega_{0}} \\
1, & x \in \overline{\Omega_{1}} \\
w_{n}(x), & x \in \bar{T}
\end{array}\right.
$$

$\tilde{w}$ is continuous by construction. Moreover, its energy is still lower than the energy of $v$ :

$$
\int_{\Omega}|\nabla \tilde{w}|^{2}=\int_{T}\left|\nabla w_{n}\right|^{2}<\int_{T}|\nabla \bar{w}|^{2}+\frac{\varepsilon}{2} \leq \int_{\Omega}|\nabla \bar{w}|^{2}+\frac{\varepsilon}{2}<\int_{\Omega}|\nabla v|^{2} .
$$

Finally, the level sets of $\tilde{w}$ are at least as big as the level sets of $v$, i.e.

$$
|\{x \in \Omega \mid \tilde{w}(x)=0\}| \geq \alpha \text { and }|\{x \in \Omega \mid \tilde{w}(x)=1\}| \geq \beta
$$

To ease notation, we will write $w$ instead of $\tilde{w}$ in what follows.

The $L^{\infty}$-constraint obviously forbids $w$ to take a value of one where $v$ is zero and vice versa, in other words:

$$
w>0 \text { on }(0, \beta) \times(0,1) \text { and } w<1 \text { on }(1-\alpha, 1) \times(0,1)
$$




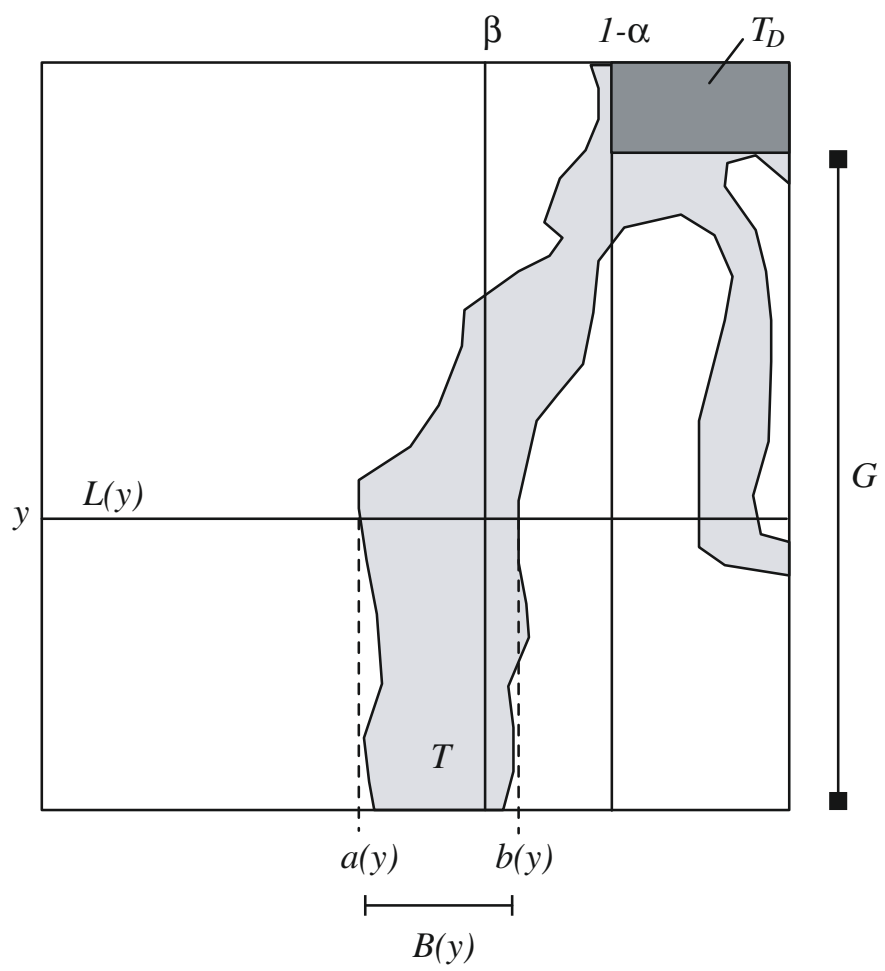

Figure 10. Illustration of the sets $T, T_{D}$ and $G$, the lines $L(y)$ and the maximal transitions from $a(y)$ to $b(y)$ with width $B(y)=|a(y)-b(y)|$.

We define $L(y):=(0,1) \times\{y\}$ and $T:=\{x \in \Omega \mid w(x) \in(0,1)\}$ (the transition layer of $w$ ), then with (3.9)

$$
\int_{0}^{1}|L(y) \cap\{(x, y) \in \Omega \mid w(x, y) \in(0,1)\}| \mathrm{d} y=|T| \leq|\Omega|-\alpha-\beta=\gamma .
$$

We denote

$$
G:=\{y \in(0,1) \mid L(y) \cap\{w=0\} \neq \emptyset \text { and } L(y) \cap\{w=1\} \neq \emptyset\}
$$

and define on $G$ the functions

$$
B(y):=\max \{|a-b| \mid w(a, y)=0, w(b, y)=1, w(t, y) \in(0,1) \text { for all } t \in(a, b)\}
$$

and $a(y), b(y)$ as the values of $a$ and $b$ maximizing $|a-b|$ in the above definition of $B(y)$.

In other words: $B(y)$ is the maximal width of a transition between zero and one on the line $L(y)$ and the boundary points of this transition are given by $(a(y), y)$ and $(b(y), y)$, compare Figure 10 for an illustration.

If we integrate over all such maximal transitions, we get a lower bound for the total area of the transition layer:

$$
\int_{G} b(y) \mathrm{d} y \leq|T| .
$$


We estimate the gradient of $w$ by its partial derivative in $x$-direction, as we did in (3.7), to get the following estimate:

$$
\begin{aligned}
\int_{\Omega}|\nabla w|^{2} & =\int_{T}|\nabla w|^{2} \geq \int_{G} \int_{0}^{1}|\nabla w(x, y)|^{2} \mathrm{~d} x \mathrm{~d} y \\
& \geq \int_{G} \int_{0}^{1}\left|\frac{\partial}{\partial x} w(x, y)\right|^{2} \mathrm{~d} x \mathrm{~d} y .
\end{aligned}
$$

Now, instead of integrating from 0 to 1 , we just integrate over the largest transition layer, i.e. from $a(y)$ to $b(y)$. We recall that $|a(y)-b(y)|=B(y)$. Using Jensen's inequality on the inner integral, we obtain therefore

$$
\int_{\Omega}|\nabla w|^{2} \geq \int_{G} \frac{1}{B(y)} \mathrm{d} y .
$$

This estimate is only useful if we find a relation between $B$ and the set $G$. Otherwise, we can choose the set $G$ small or $B$ large to reduce the energy. Therefore we want to estimate the size of $G$. Let us define some area of the transition layer $T$ that is situated outside $(0,1) \times G$ by

$$
T_{D}:=(0,1) \times((0,1) \backslash G) \cap T,
$$

compare again Figure 10 where this set is shaded in dark grey. Let $\delta:=\left|T_{D}\right|$ be the size of this area.

Since for $y \in(0,1) \backslash G$ we cannot have $w\left(x_{1}, y\right)=0$ and $w\left(x_{2}, y\right)=1$ for two values $x_{1}, x_{2} \in(0,1)$, and on the other hand $w(x, y)<1$ for $x>1-\alpha$ and $w(x, y)>0$ for $x<\beta$, see (3.10), we need to "cover" either $(0, \beta) \times((0,1) \backslash G)$ or $(1-\alpha, 1) \times((0,1) \backslash G)$ by the transition layer. Thus we get a lower bound for $\delta$ (taking into account that $\alpha<\beta)$ :

$$
\delta \geq \alpha(1-|G|)
$$

Resolved for $G$, we obtain

$$
|G| \geq 1-\frac{\delta}{\alpha}
$$

Now we can continue estimating the energy of $w$. We first apply the Jensen inequality with $\bar{B}$ being the average over $B$ on $G$ :

$$
\int_{\Omega}|\nabla w|^{2} \geq \int_{G} \frac{1}{B(y)} \mathrm{d} y \geq|G| \frac{1}{\bar{B}} .
$$

Let $T_{G}:=\left.T\right|_{(0,1) \times G}$ be the transition layers on $(0,1) \times G$. Since $T_{G} \cup T_{D} \subset T$ and $T_{G}$ and $T_{D}$ are disjoint, we have $\left|T_{G}\right| \leq|T|-\left|T_{D}\right|$. Using that $\delta=\left|T_{D}\right|$ and that $|T| \leq \gamma$, we have $\left|T_{G}\right| \leq \gamma-\delta$.

On the other hand, $\int_{G} B(y) \mathrm{d} y \leq\left|T_{G}\right|$, thus $\bar{B}|G| \leq \gamma-\delta$ or in other words $\bar{B} \leq(\gamma-\delta) /|G|$. This provides us with the necessary relation between $B$ and the size of $G$. Together with (3.12) we obtain

$$
\int_{\Omega}|\nabla w|^{2} \geq|G|^{2} \frac{1}{\gamma-\delta} .
$$

Inserting (3.11), gives

$$
\int_{\Omega}|\nabla w|^{2} \geq \frac{(1-\delta / \alpha)^{2}}{\gamma-\delta}
$$


We calculate the difference between this energy and the energy of $v$, as computed in (3.7):

$$
\begin{aligned}
\int_{\Omega}|\nabla w|^{2}-\int_{\Omega}|\nabla v|^{2} & \geq \frac{(1-\delta / \alpha)^{2}}{\gamma-\delta}-\frac{1}{\gamma}=\frac{-2 \frac{\delta}{\alpha} \gamma+\frac{\delta^{2}}{\alpha} \gamma+\delta}{\gamma(\gamma-\delta)} \\
& \geq \frac{\delta}{\gamma(\gamma-\delta)}\left(1-2 \frac{\gamma}{\alpha}\right) .
\end{aligned}
$$

Using (3.6), we see that the right hand side is larger or equal than zero. This proves that $w$ cannot have a smaller energy than $v$, thus $v$ is a local minimizer.

Acknowledgements. We thank Giuseppe Buttazzo for his suggestions which helped to initiate this work.

\section{REFERENCES}

[1] E. Acerbi, I. Fonseca and G. Mingione, Existence and regularity for mixtures of micromagnetic materials. Proc. Royal Soc. London Sect. A 462 (2006) 2225-2244.

[2] N. Aguilera, H.W. Alt and L.A. Caffarelli, An optimization problem with volume constraint. SIAM J. Control Optim. 24 (1986) 191-198.

[3] G. Allaire, F. Jouve and A.M. Toader, A level-set method for shape optimization. C. R. Acad. Sci. Paris 334 (2002) 1125-1130.

[4] L. Ambrosio, I. Fonseca, P. Marcellini and L. Tartar, On a volume-constrained variational problem. Arch. Ration. Mech. Anal. 149 (1999) 23-47.

[5] A. Braides, $\Gamma$-convergence for beginners, Oxford Lecture Series in Mathematics and its Applications 22. Oxford University Press, Oxford (2002).

[6] D. Bucur and G. Buttazzo, Variational Methods in Shape Optimization Problems 65. Birkhäuser Boston (2005).

[7] G. Dal Maso, An Introduction to Г-Convergence. Birkhäuser Boston Inc., Boston, MA (1993).

[8] M.E. Gurtin, D. Polignone and J. Vinals, Two-phase binary fluids and immissible fluids described by an order parameter. Math. Models Methods Appl. Sci. 6 (1996) 815-831.

[9] A. Henrot and M. Pierre, Variation et optimisation de formes, une analyse géométrique 48. Springer-Verlag, Paris (2005).

[10] M. Morini and M.O. Rieger, On a volume constrained variational problem with lower order terms. Appl. Math. Optim. 48 (2003) 21-38.

[11] S. Mosconi and P. Tilli, Variational problems with several volume constraints on the level sets. Calc. Var. Part. Diff. Equ. 14 (2002) 233-247.

[12] S. Osher and F. Santosa, Level set methods for optimization problems involving geometry and constraints: frequencies of a two-density inhomogeneous drum. J. Comput. Phys 171 (2001) 272-288.

[13] S. Osher and J.A. Sethian, Front propagation with curvature-dependant speed: Algorithms based on Hamilton-Jacobi formulations. J. Comput. Phys. 79 (1988) 12-49.

[14] E. Oudet, Numerical minimization of eigenmodes of a membrane with respect to the domain. ESAIM: COCV 10 (2004) 315-335.

[15] M.O. Rieger, Abstract variational problems with volume constraints. ESAIM: COCV 10 (2004) 84-98.

[16] M.O. Rieger, Higher dimensional variational problems with volume constraints - existence results and $\Gamma$-convergence. Interfaces and Free Boundaries (to appear).

[17] J. Sokolowski and J.P. Zolesio, Introduction to Shape Optimization: Shape Sensitivity Analysis, Springer Series in Computational Mathematics 10. Springer (1992).

[18] P. Vergilius Maro, Aeneidum I. (29-19 BC). 